\title{
Predicted vitamin D status and incidence of tooth loss and periodontitis
}

\author{
Monik Jimenez ${ }^{1}$, Edward Giovannucci ${ }^{2}$, Elizabeth Krall Kaye ${ }^{3}$, Kaumudi J Joshipura ${ }^{4}$ \\ and Thomas Dietrich ${ }^{5, *}$ \\ 'Division of Preventive Medicine, Brigham and Women's Hospital, Boston, MA, USA: ${ }^{2}$ Department of Nutrition, \\ Harvard School of Public Health, Boston, MA, USA: ${ }^{3}$ Department of Health Policy and Health Services Research, \\ Boston University Henry M. Goldman School of Dental Medicine, Boston, MA, USA: ${ }^{4}$ University of Puerto Rico \\ School of Dentistry, San Juan, Puerto Rico: ${ }^{5}$ Department of Oral Surgery, The School of Dentistry, University of \\ Birmingham, St Chad's Queensway, Birmingham B4 6NN, UK
}

Submitted 2 August 2012: Final revision received 19 November 2012: Accepted 25 November 2012: First published online 7 March 2013

\begin{abstract}
Objective: Vitamin D insufficiency is highly prevalent, with particular subgroups at greater risk (e.g. the elderly and those with darker skin). Vitamin D insufficiency may partly explain US racial/ethnic disparities in the prevalence of periodontitis and tooth loss. We evaluated the association between a predictor score of plasma 25-hydroxyvitamin D (25(OH)D) and incidence of periodontitis and tooth loss. Design: Detailed biennial questionnaires were collected on medical history, lifestyle practices and incident periodontitis and tooth loss. The predictor score was derived from variables known to influence circulating concentrations of plasma $25(\mathrm{OH}) \mathrm{D}$ and validated against plasma concentrations among a sub-sample. Multivariable Cox proportional-hazards models with time-varying covariates estimated the association between the predicted $25(\mathrm{OH}) \mathrm{D}$ score and time until first tooth loss. Subjects: A total of 42730 participants of the Health Professionals Follow-Up Study aged 40-75 years at baseline were followed from 1986 to 2006.

Setting: USA, representing all fifty states and the District of Columbia.

Results: We observed 13581 incident tooth loss events from 539335 person-years. There was a dose-dependent significant inverse association across quintiles of the predicted $25(\mathrm{OH}) \mathrm{D}$ score and incidence of tooth loss. In multivariable analyses, the highest quintile of the updated predicted $25(\mathrm{OH}) \mathrm{D}$ score compared with the lowest was associated with a $20 \%$ lower incidence of tooth loss (hazard ratio $=0 \cdot 80$, 95\% CI 0.76, 0.85; $P$ value for trend $<0.001)$; UV-B was also independently associated. Results for the predicted $25(\mathrm{OH}) \mathrm{D}$ score and periodontitis were similar. Conclusions: These results are suggestive of an association between predictors of vitamin D and lower incidence of tooth loss and periodontitis.
\end{abstract}

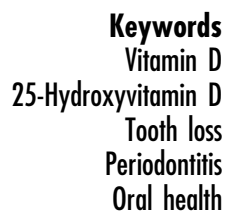

Keywords

vitamin D

Oral health
Studies have suggested that vitamin D may increase bone mineral density and reduce fracture risk over a wide range of serum 25-hydroxyvitamin D (25(OH)D) concentrations. The serum concentration of $25(\mathrm{OH}) \mathrm{D}$ that would define adequate vitamin D status with respect to bone health is currently estimated to be at least $75 \mathrm{nmol} / \mathrm{l}^{(1)}$. Data from observational studies suggest that serum $25(\mathrm{OH}) \mathrm{D}$ concentrations of at least $75 \mathrm{nmol} / 1$ may also be beneficial for other health outcomes such as colorectal and prostate cancer $^{(2,3)}$, diabetes ${ }^{(4)}$ and possibly gingivitis and periodontitis ${ }^{(5,6)}$

Unfortunately, a majority of the US population has vitamin D insufficiency with particular subgroups of the population at an even greater risk (e.g. the elderly and those with dark skin) even among otherwise healthy adults $^{(7,8)}$. Moreover, considerable differences in 25(OH)D serum concentration exist between racial/ethnic groups in the USA, most strikingly between non-Hispanic whites and non-Hispanic blacks. In a representative survey of the US population, the third National Health and Nutrition Examination Survey (1988-1994) reported that significantly more non-Hispanic blacks had serum 25(OH)D concentration below $50 \mathrm{nmol} / \mathrm{l}$ than did non-Hispanic whites $^{(9)}$. Obesity may also play a key role in the association between vitamin D status and tooth loss. Non-Hispanic blacks and Mexican Americans exhibit a higher prevalence of obesity compared with non-Hispanic whites ${ }^{(10)}$ and obesity has been shown to alter the production of $25(\mathrm{OH}) \mathrm{D}^{(11)}$. These marked differences in vitamin $\mathrm{D}$ status may, in part, explain the racial/ethnic disparities in prevalence of periodontitis and tooth loss observed in the USA. 
We hypothesized that lower serum concentrations of $25(\mathrm{OH}) \mathrm{D}$ would be associated with an increased rate of self-reported periodontitis and tooth loss. Therefore, we aimed to evaluate whether vitamin D status was associated with the incidence of tooth loss and selfreported periodontitis, using prospective data from the Health Professionals Follow-up Study (HPFS).

\section{Methods}

\section{Study participants}

The HPFS consists of 51529 male health professionals aged 40-75 years, from across all US states and the District of Columbia, who completed a mailed questionnaire in 1986 after providing informed consent to participate ${ }^{(12)}$. The cohort was initiated to explore associations between diet, heart disease and cancer ${ }^{(12)}$. The initial questionnaire collected data regarding diet, lifestyle behaviours, physical characteristics (including height and weight), medication use, and medical and dental history. Follow-up questionnaires have been mailed biennially since 1988 collecting data on various exposures and outcomes and a detailed semi-quantitative FFQ has been mailed every 4 years. Over $90 \%$ of the baseline population has responded to follow-up questionnaires $^{(13)}$.

The HPFS was conducted according to the guidelines laid down in the Declaration of Helsinki and all procedures involving human subjects/patients were approved by the Institutional Review Board of Boston University Henry M. Goldman School of Dental Medicine and were in accordance with institutional guidelines. Written informed consent was obtained from all subjects/patients. The present analysis was approved by the Board for the Protection of Human Subjects at Boston University Henry M. Goldman School of Dental Medicine.

Participants were excluded from the tooth loss analysis if they were missing data on baseline number of teeth, did not wish to participate in the study, were edentulous at baseline, had a previous diagnosis of cancer at baseline, provided poor dietary data or were missing data on region of residence; additionally men were excluded at baseline due to missing data on smoking, BMI and physical activity but were allowed to re-enter analyses in later cycles, resulting in a final sample of 42730 men. Men were additionally excluded from the periodontitis analysis if they reported a history of periodontitis at baseline, resulting in a final sample of 35644 .

\section{Covariate ascertainment}

The baseline and follow-up questionnaires collected detailed information on smoking status, including average number of cigarettes smoked per day, smoking duration and, for former smokers, time since cessation. These data were used to calculate a comprehensive smoking index (CSI) as previously described ${ }^{(14,15)}$. Furthermore, data on dental profession, race and region of residence were collected at baseline and data regarding chewing tobacco use were collected in 1996. Data on routine physical examination in the last 2 years and pipe use were collected on each biennial questionnaire. Alcohol use, multivitamin use, vitamins $\mathrm{E}$ and $\mathrm{C}$ supplementation and dietary factors were assessed from a quadrennial semi-quantitative FFQ which has been validated against diet records with high validity ${ }^{(16,17)}$. Self-reported binary diabetes status has been collected on all biennial questionnaires and previously validated against medical records in a sub-sample of men demonstrating excellent validity, with $97 \%$ of cases confirmed ${ }^{(18)}$. Self-reported BMI was calculated from height (collected in 1986) and weight (all biennial questionnaires). Measures of BMI were validated against technician measurements among a subset of participants residing in the Boston area with high validity ${ }^{(12)}$. Physical activity was based on various self-reported activities (collected on all biennial questionnaires) and converted into metabolic equivalents per week (MET/week); it has been validated previously against past-week recalls and activity diaries in a subset of men with reasonable validity for leisure-time activity ${ }^{(19)}$.

\section{Predicted 25(OH)D score}

The derivation and validation of the multivariable prediction score used to estimate predicted $25(\mathrm{OH}) \mathrm{D}$ plasma concentration based on known predictors of circulating plasma $25(\mathrm{OH}) \mathrm{D}^{(20)}$, hereafter referred to as 'predicted 25(OH)D score', has been described in detail previously $^{(20)}$. Briefly, the predicted $25(\mathrm{OH}) \mathrm{D}$ score was developed among a sub-sample of 1095 men from the HPFS who had plasma available and were free of cancer at the time of blood collection. Dietary and lifestyle factors were similar among men who provided blood samples and those who did not. Linear regression models were estimated between plasma 25(OH)D (dependent variable) and predictors of plasma $25(\mathrm{OH}) \mathrm{D}$ (independent variables), which were determined based on subject matter knowledge. A predicted $25(\mathrm{OH}) \mathrm{D}$ score was estimated for each participant based on estimated regression coefficients. The predictor score was estimated from each of the following components: leisure-time physical activity (quintiles of MET/week), BMI $(<22 \cdot 0,22 \cdot 0-24 \cdot 9,25 \cdot 0-29 \cdot 9$, $\left.30 \cdot 0-34 \cdot 9, \geq 35 \cdot 0 \mathrm{~kg} / \mathrm{m}^{2}\right)$, vitamin $\mathrm{D}$ from food $(<2 \cdot 50$, $2 \cdot 50-4.99,5 \cdot 00-7 \cdot 49,7 \cdot 50-9 \cdot 99, \geq 10 \cdot 00 \mu \mathrm{g} / \mathrm{d})$, vitamin D from supplement sources (0, 0.025-4.99, 5.00-9.99, $\geq 10 \cdot 00 \mu \mathrm{g} / \mathrm{d}$ ), race (white, African American, Asian, other) and UV-B flux $\left(<461,461-<628, \geq 628 \mathrm{~J} / \mathrm{cm}^{2}\right)$. Race (proxy for skin pigmentation), UV-B flux (average annual UV-B flux was derived from geographic region of residence at baseline which represents a composite measure of mean UV-B radiation level accounting for latitude, altitude and cloud cover) ${ }^{(21)}$, vitamin D intake from diet, BMI and physical activity (proxy for outdoor sunlight exposure) 
were identified as independent predictors of $25(\mathrm{OH}) \mathrm{D}^{(20)}$. The difference in mean plasma $25(\mathrm{OH}) \mathrm{D}$ across extreme deciles of the predicted $25(\mathrm{OH}) \mathrm{D}$ score was $27 \cdot 8 \mathrm{nmol} / 1$. The model identified a wide range of predicted $25(\mathrm{OH}) \mathrm{D}$, ranging from a summer high of $91 \mathrm{nmol} / 1$ for a man with all favourable characteristics (i.e. highest intake categories, residence in South/Southwest, lowest BMI, highest activity level, low skin pigmentation) to a predicted winter low of $23 \mathrm{nmol} / 1$ for the opposite extreme. The predicted $25(\mathrm{OH}) \mathrm{D}$ score was then validated in a separate sample of 542 men who had plasma 25(OH)D levels available and were free of cancer at the time of blood collection. The difference in mean plasma $25(\mathrm{OH}) \mathrm{D}$ across extreme deciles of the predicted $25(\mathrm{OH}) \mathrm{D}$ score was $25.0 \mathrm{nmol} / \mathrm{l}$, which was similar to that observed in the development data set. The associations between the predicted $25(\mathrm{OH}) \mathrm{D}$ score and key demographic and dietary characteristics were as expected for plasma 25(OH)D.

\section{Outcome}

Incident tooth loss, the primary outcome of interest, was measured by self-report from biennial questionnaires beginning in 1986 through 2006. Self-reported tooth loss measures have been validated in other populations and found to be highly valid ${ }^{(22)}$. Furthermore, the HPFS population comprised health professionals with over $50 \%$ being dentists; hence we expect tooth loss to be reported with high validity.

Data on periodontitis were collected on each biennial questionnaire. The question administered was 'Have you been professionally diagnosed with periodontal disease with bone loss?' Men who responded 'Yes' were considered positive for periodontitis. Self-reported periodontitis has been validated in this population and shown good validity against bitewing radiographs among both dentists and non-dentists ${ }^{(23,24)}$. The self-reported binary measure of periodontitis has been validated against bitewing radiographs, using a case definition based on alveolar bone loss which reflects history of periodontitis. Bitewing radiographs were obtained from 352 men and classified as periodontally diseased for bone loss $>2 \mathrm{~mm}$ and/or complete loss of the crestal lamina dura ${ }^{(23,24)}$. Radiographs were reviewed by three examiners blinded to the respondents' self-reported periodontal disease status. Among dentists self-reported periodontitis status showed a positive predictive value $(\mathrm{PV}+)$ of 0.76 and a negative predictive value $(\mathrm{PV}-$ ) of $0 \cdot 74$, and among non-dentists the corresponding values were $\mathrm{PV}+=0.83$ and $\mathrm{PV}-=0.69$. Hence, the self-reported measure shows good validity in representing periodontitis compared with radiographic bone loss, which is a well-accepted clinical measure of the cumulative effects of periodontitis ${ }^{(25)}$.

\section{Statistical methods}

The analysis consisted of examining two relationships: (i) the association between predicted $25(\mathrm{OH}) \mathrm{D}$ score over follow-up and incident tooth loss; and (ii) the association between predicted $25(\mathrm{OH}) \mathrm{D}$ score over follow-up and incident periodontitis. Cox models ${ }^{(26)}$ were used to estimate the association between time-varying predicted $25(\mathrm{OH}) \mathrm{D}$ score and incident tooth loss and periodontitis, separately, using age- and multivariable-adjusted models. The predicted $25(\mathrm{OH}) \mathrm{D}$ score was updated at each follow-up cycle based on updated values for each of the components as follows: physical activity and BMI were updated biennially, vitamin D from foods and supplements were updated every 4 years, whereas race and UV-B radiation were based on a single measurement at baseline in 1986. The effect estimates presented are hazard ratios (HR) with corresponding 95\% confidence intervals. Person-time was calculated from the date of return of the baseline questionnaire in 1986 to the date of first self-reported tooth loss/periodontitis, date of death, diagnosis of cancer or 31 January 2006, whichever came first. To address missing information, data were carried forward no more than one questionnaire cycle, the median value was substituted for missing values on the CSI variable and the complete case method was utilized thereafter $(<5 \%$ of observations were missing data on any particular covariate).

Potential confounders and risk factors were selected based on a priori subject matter knowledge, the underlying causal structure of the association between vitamin $\mathrm{D}$, tooth loss and periodontitis, and examination of a $\geq 10 \%$ change in main effect estimates; however, covariates deemed clinically significant were retained irrespective of the $10 \%$ change in estimate criterion. Two multivariable analyses were conducted for each outcome. The primary analyses are provided in Model 1 adjusted for smoking history (CSI), pipe or chewing tobacco use (yes/no), multivitamin use (yes/no), vitamin E supplementation (quintiles), vitamin $\mathrm{C}$ supplementation (quintiles), dental professional (yes/no), alcohol use $(0,0 \cdot 1-4 \cdot 9,5 \cdot 0-14 \cdot 9$, $15 \cdot 0-29 \cdot 9, \geq 30 \cdot 0 \mathrm{~g} / \mathrm{d})$, routine physical examination (yes/no) and diabetes status (yes/no). Model 2 presents sensitivity analyses further adjusted for components of the predicted $25(\mathrm{OH}) \mathrm{D}$ score, including $\mathrm{BMI}(<22 \cdot 0$, $\left.22 \cdot 0-24 \cdot 9, \quad 25 \cdot 0-29 \cdot 9, \quad 30 \cdot 0-34 \cdot 9, \quad \geq 35 \cdot 0 \mathrm{~kg} / \mathrm{m}^{2}\right)$, race (white, African American, Asian, other) and physical activity (quintiles). We additionally evaluated the association between the components used to create the predicted $25(\mathrm{OH}) \mathrm{D}$ score and tooth loss in a model without the predicted $25(\mathrm{OH}) \mathrm{D}$ score while adjusting for all other confounders described in multivariable Model 1.

A priori we proposed to evaluate whether the association between the predicted $25(\mathrm{OH}) \mathrm{D}$ score and tooth loss or periodontitis varied by age, BMI, dental occupation, physical activity and alcohol intake, based on subject matter knowledge. The variation of these associations across the a priori identified covariates was evaluated by the introduction of interaction terms into a model with continuous main effects (multivariable Model 1). 
Significance of the interaction was evaluated by a likelihood ratio test. We evaluated the influence of misclassification of the outcome on effect estimates using methods proposed by Duffy et al. ${ }^{(27)}$. Predictive values were obtained from the prior validation studies conducted by Joshipura et $a l^{(23,24)}$ separately among independent samples of dentists and non-dentists from this cohort. A weighted average of these independent samples was calculated to obtain mean predictive values $\left(\mathrm{PV}_{\mathrm{m}+}\right.$ and $\left.\mathrm{PV}_{\mathrm{m}-}\right)$. The adjusted HR was estimated by taking the exponential of the $\log$ of the uncorrected HR divided by the sum of $\left(\mathrm{PV}_{\mathrm{m}+}, \mathrm{PV}_{\mathrm{m}-}\right.$ and -1$)$. All $P$ values were two-sided at $\alpha=0 \cdot 05$. Analyses were conducted with the SAS for UNIX statistical software package version $9 \cdot 1 \cdot 3$.

\section{Results}

Over 20 years of follow-up 13581 men reported incident tooth loss and 3158 men reported incident periodontitis. Men within the highest quintile of the predicted 25(OH)D score were more likely to be Caucasian, physically active, live in the South/Southwest and less likely to be diabetic or report periodontitis compared with those in the lowest quintile of the predicted $25(\mathrm{OH}) \mathrm{D}$ score (Table 1 ). As expected, men in the highest quintile of the predicted $25(\mathrm{OH}) \mathrm{D}$ score consumed more vitamin D from food and supplements than those in the lowest quintile.

The predicted $25(\mathrm{OH}) \mathrm{D}$ score was associated with a lower risk of incident tooth loss among men in age- and multivariable-adjusted models (Table 2). Men with a predicted $25(\mathrm{OH}) \mathrm{D}$ score in the highest quintile exhibited a significantly lower risk of tooth loss compared with men in the lowest quintile after adjustment for covariates in multivariable Model 1 (smoking, pipe, chewing tobacco, multivitamin use, vitamin $\mathrm{E}$, vitamin $\mathrm{C}$, dental profession, alcohol consumption, routine physical examination and diabetes status). When additionally adjusted for race, BMI and physical activity (Model 2), the association was slightly attenuated. Each $10 \mathrm{nmol} / 1$ increase in the predicted $25(\mathrm{OH}) \mathrm{D}$ score was associated with a $10 \%$ significantly lower risk of tooth loss when adjusted for confounders in Model 1; the results were similar when additionally adjusted for race, BMI and physical activity (Model 2). We separately evaluated the influence of $\mathrm{Ca}$ intake on multivariable association between quintiles of the predicted $25(\mathrm{OH}) \mathrm{D}$ score and tooth loss (Model 1); however, estimates were materially unchanged (results not shown).

Table 1 Age-standardized baseline characteristics by quintile of the predicted $25(\mathrm{OH}) \mathrm{D}$ score* among 42730 men participating in the Health Professionals Follow-up Study, USA, 1986-2006

\begin{tabular}{|c|c|c|c|c|c|c|}
\hline & \multicolumn{2}{|c|}{ Q1 } & \multicolumn{2}{|c|}{ Q3 } & \multicolumn{2}{|c|}{ Q5 } \\
\hline & Mean or \% & SD & Mean or $\%$ & SD & Mean or \% & SD \\
\hline$\%$ & 20 & - & 20 & & 20 & \\
\hline$n$ & 8513 & & 8308 & & 8704 & \\
\hline Age (years) & 54 & $9 \cdot 6$ & 54 & $9 \cdot 7$ & 54 & $9 \cdot 8$ \\
\hline \multicolumn{7}{|l|}{ Race (\%) } \\
\hline Caucasian & 87 & - & 96 & - & 97 & - \\
\hline African American & 4 & - & $0 \cdot 1$ & - & - & - \\
\hline Asian & 6 & - & 0.9 & - & $0 \cdot 1$ & - \\
\hline BMI $\left(\mathrm{kg} / \mathrm{m}^{2}\right)$ & $27 \cdot 2$ & $3 \cdot 6$ & $25 \cdot 5$ & $3 \cdot 2$ & $23 \cdot 7$ & $2 \cdot 4$ \\
\hline \multicolumn{7}{|l|}{ Smokers (\%) } \\
\hline Never & 45 & - & 48 & - & 52 & - \\
\hline Current & 12 & - & 9 & - & 6 & - \\
\hline Former & 43 & - & 43 & - & 42 & - \\
\hline Diabetes (\%) & 4 & - & 3 & - & 3 & - \\
\hline Alcohol $(\mathrm{g} / \mathrm{d})$ & $11 \cdot 7$ & $17 \cdot 0$ & $11 \cdot 3$ & $15 \cdot 4$ & $11 \cdot 0$ & $13 \cdot 9$ \\
\hline Physical activity (MET/week) & $5 \cdot 0$ & $7 \cdot 6$ & $18 \cdot 4$ & $22 \cdot 5$ & $42 \cdot 4$ & 38.9 \\
\hline Dentist (\%) & 53 & - & 58 & - & 65 & - \\
\hline Married (\%) & 91 & - & 91 & - & 88 & - \\
\hline \multicolumn{7}{|l|}{ Region (\%) } \\
\hline Northeast/Mid-Atlantic & 26 & - & 23 & - & 16 & - \\
\hline South/Southwest & 36 & - & 42 & - & 59 & - \\
\hline Midwest/Pacific Northwest & 37 & - & 35 & - & 24 & - \\
\hline Periodontitis $(\%)+$ & 17 & - & 16 & - & 14 & - \\
\hline \multicolumn{7}{|l|}{ Number of teeth (\%) } \\
\hline $25-32$ & 81 & - & 84 & - & 87 & - \\
\hline $17-24$ & 13 & - & 12 & - & 10 & - \\
\hline $11-16$ & 3 & - & 2 & - & 2 & - \\
\hline $1-10$ & 2 & - & 2 & - & 1 & - \\
\hline Vitamin D from food $(\mu \mathrm{g} / \mathrm{d})$ & 4.53 & $2 \cdot 55$ & $6 \cdot 48$ & $3 \cdot 55$ & $8 \cdot 45$ & $4 \cdot 05$ \\
\hline Vitamin D from supplements $(\mu \mathrm{g} / \mathrm{d})$ & $1 \cdot 70$ & $4 \cdot 5$ & 3.53 & $6 \cdot 20$ & $5 \cdot 43$ & $7 \cdot 05$ \\
\hline
\end{tabular}

25(OH)D, 25-hydroxyvitamin D, Q1, lowest quintile; Q3, middle quintile; Q5, highest quintile; MET, metabolic equivalents.

${ }^{*}$ Values are standardized to the age distribution of the study population. Percentages may not sum to $100 \%$ due to rounding.

tMen with periodontitis at baseline were excluded from the incident periodontitis analysis. 
Table 2 Age- and multivariate-adjusted hazard ratios and $95 \%$ confidence intervals for the predicted 25(OH)D score and incident tooth loss and periodontitis among men participating in the Health Professionals Follow-up Study, USA, 1986-2006

\begin{tabular}{|c|c|c|c|c|c|c|c|c|c|}
\hline \multirow{2}{*}{$\begin{array}{l}\text { Predicted } \\
25(\mathrm{OH}) \text { D score }\end{array}$} & \multirow[b]{2}{*}{ Events } & \multirow{2}{*}{$\begin{array}{l}\text { Person- } \\
\text { years }\end{array}$} & \multirow{2}{*}{$\begin{array}{l}\text { Incidence } \\
\text { rate } \neq\end{array}$} & \multicolumn{2}{|c|}{ Age adjusted } & \multicolumn{2}{|c|}{ Model $1^{*}$} & \multicolumn{2}{|c|}{ Model 2† } \\
\hline & & & & $\mathrm{HR}$ & $95 \% \mathrm{Cl}$ & HR & $95 \% \mathrm{Cl}$ & $H R$ & $95 \% \mathrm{Cl}$ \\
\hline \multicolumn{10}{|l|}{ Tooth loss ( $n$ 42730) } \\
\hline Continuous $\$$ & 13581 & 539335 & 2518 & $0 \cdot 86$ & $0 \cdot 84,0.88$ & 0.90 & $0.88,0.92$ & 0.93 & $0.90,0.97$ \\
\hline \multicolumn{10}{|l|}{ Quintiles } \\
\hline Q1 (ref.) & 2879 & 100650 & 2860 & $1 \cdot 00$ & - & 1.00 & - & 1.00 & - \\
\hline Q2 & 2796 & 104191 & 2684 & 0.91 & $0.86,0.96$ & 0.93 & $0.88,0.98$ & 0.97 & $0.91,1.02$ \\
\hline Q3 & 2728 & 107687 & 2533 & $0 \cdot 86$ & $0.81,0.90$ & 0.90 & $0 \cdot 85,0.94$ & 0.94 & $0.88,1.00$ \\
\hline Q4 & 2617 & 111088 & 2356 & 0.78 & $0.74,0.82$ & $0 \cdot 84$ & $0.79,0.89$ & 0.89 & $0.83,0.95$ \\
\hline Q5 & 2561 & 115720 & 2213 & 0.72 & $0.68,0.76$ & $0 \cdot 80$ & $0.76,0.85$ & 0.86 & $0.79,0.93$ \\
\hline$P$ value for trend & & & & \multicolumn{2}{|c|}{$<0.001$} & \multicolumn{2}{|c|}{$<0.001$} & \multicolumn{2}{|c|}{0.001} \\
\hline \multicolumn{10}{|l|}{ Periodontitis ( $n 35644)$} \\
\hline Continuous $\S$ & 3158 & 547248 & 577 & $0 \cdot 88$ & $0.84,0.92$ & 0.91 & $0.86,0.95$ & $1 \cdot 01$ & $0.93,1.10$ \\
\hline \multicolumn{10}{|l|}{ Quintiles } \\
\hline Q1 (ref.) & 673 & 102935 & 654 & $1 \cdot 00$ & - & $1 \cdot 00$ & - & $1 \cdot 00$ & - \\
\hline Q2 & 629 & 105771 & 595 & 0.91 & $0.81,1.01$ & 0.92 & $0.82,1.03$ & 0.98 & $0.87,1 \cdot 11$ \\
\hline Q3 & 638 & 109436 & 583 & 0.88 & $0.79,0.98$ & 0.91 & $0 \cdot 81,1 \cdot 01$ & $1 \cdot 01$ & $0 \cdot 88,1 \cdot 15$ \\
\hline Q4 & 639 & 112538 & 568 & 0.85 & $0.76,0.95$ & $0 \cdot 88$ & $0.79,0.99$ & $1 \cdot 02$ & $0 \cdot 88,1 \cdot 18$ \\
\hline Q5 & 579 & 116568 & 497 & 0.74 & $0.66,0.83$ & $0 \cdot 80$ & $0.71,0.90$ & 0.97 & $0 \cdot 81,1 \cdot 16$ \\
\hline$P$ value for trend & & & & \multicolumn{2}{|c|}{$<0.0001$} & \multicolumn{2}{|c|}{$<0.001$} & \multicolumn{2}{|c|}{0.89} \\
\hline
\end{tabular}

25(OH)D, 25-hydroxyvitamin D; HR, hazard ratio; Q1-Q5, quintile 1-quintile 5; ref., reference category; MET, metabolic equivalents.

${ }^{*}$ Model 1: age, smoking (CSI, comprehensive smoking index), pipe (yes/no), chewing tobacco (yes/no), multivitamin use (yes/no), vitamin E (quintiles), vitamin

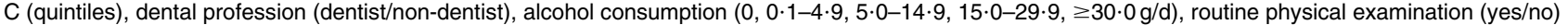
and diabetes (yes/no).

tModel 2: Model $1+$ BMI (<22.0 (ref.), 22.0-24.9, 25.0-29.9, 30.0-34.9, $\geq 35 \cdot 0 \mathrm{~kg} / \mathrm{m}^{2}$ ), race (white, African American, Asian, other), and physical activity (MET/week; quintiles).

‡Incidence rate per 100000 person-years.

$\S$ Per $10 \mathrm{nmol} / \mathrm{l}$.

There was evidence to suggest that the association between the predicted $25(\mathrm{OH}) \mathrm{D}$ score and tooth loss varied by age and dental profession (Fig. 1). The association between the predicted 25(OH)D score and tooth loss was stronger among younger men compared with the association observed among men aged $\geq 65$ years ( $P$ value for interaction $=0 \cdot 03$ ). Furthermore, the association between the predicted $25(\mathrm{OH}) \mathrm{D}$ score and tooth loss among dentists was stronger than the association observed among non-dentists $(P$ value for interaction $=0 \cdot 01$ ). We additionally modelled quintiles of the predicted $25(\mathrm{OH}) \mathrm{D}$ score among dentists and observed that when compared with the lowest quintile, all higher quintiles of the predicted $25(\mathrm{OH}) \mathrm{D}$ score were significantly associated with a lower risk of tooth loss (reference $=\mathrm{Q} 1 ; \mathrm{Q} 2: \mathrm{HR}=0.89(95 \%$ CI 0.82, 0.96); Q3: $\mathrm{HR}=0.86(95 \%$ CI 0.80, 0.93); Q4: $\mathrm{HR}=0.80(95 \%$ CI $0.74,0.87) ; \mathrm{Q} 5: \mathrm{HR}=0.73(95 \%$ CI $0.68,0.79)$ ). There were no significant interactions observed for the predicted $25(\mathrm{OH}) \mathrm{D}$ score and periodontitis with any of the covariates in Fig. 1 (results not shown).

We additionally evaluated the association between the components of the predicted 25(OH)D score and tooth loss without including the predicted $25(\mathrm{OH}) \mathrm{D}$ score while adjusting for all potential confounders in Model 1 (Table 3). We found a significantly lower risk of tooth loss associated with residence in an area with UV-B flux $\geq 628 \mathrm{~J} / \mathrm{cm}^{2}$ when compared with the UV-B flux $<461 \mathrm{~J} / \mathrm{cm}^{2}$; and an increased risk of tooth loss with BMI $\geq 35 \cdot 0 \mathrm{~kg} / \mathrm{m}^{2}$ compared with $\mathrm{BMI}<22 \cdot 0 \mathrm{~kg} / \mathrm{m}^{2}$. There was no association observed between tooth loss and vitamin $\mathrm{D}$ intake from food $(<2.50 \mu \mathrm{g} / \mathrm{d} v$. $\geq 10 \cdot 00 \mu \mathrm{g} / \mathrm{d})$ or supplements $(0 \mu \mathrm{g} / \mathrm{d} v . \geq 10 \cdot 00 \mu \mathrm{g} / \mathrm{d})$. An elevated but non-significant association was observed between African American race and tooth loss compared with Caucasians.

Overall, results were similar for periodontitis (Table 2). When adjusting for covariates in Model 1, the highest quintile of the predicted $25(\mathrm{OH}) \mathrm{D}$ score was associated with a lower risk of periodontitis compared with the lowest quintile. Additionally adjusting for components of the predictor score (Model 2) resulted in substantial attenuation of the association towards the null. When evaluated as a continuous exposure, the risk of periodontitis decreased significantly by $9 \%$ for each $10 \mathrm{nmol} / 1$ increase in the predicted $25(\mathrm{OH}) \mathrm{D}$ score, when adjusted for potential confounders in Model 1.

\section{Discussion}

The present study is the longest prospective observational study to evaluate the association between predictors of $25(\mathrm{OH}) \mathrm{D}$ and incidence of tooth loss and periodontitis. In multivariable analyses, adjusted for key confounders and risk factors, the risk of tooth loss and periodontitis was significantly lower with higher levels of the predicted $25(\mathrm{OH}) \mathrm{D}$ score. Furthermore, the association between the predicted $25(\mathrm{OH}) \mathrm{D}$ score and tooth loss may vary by age and dental profession. 


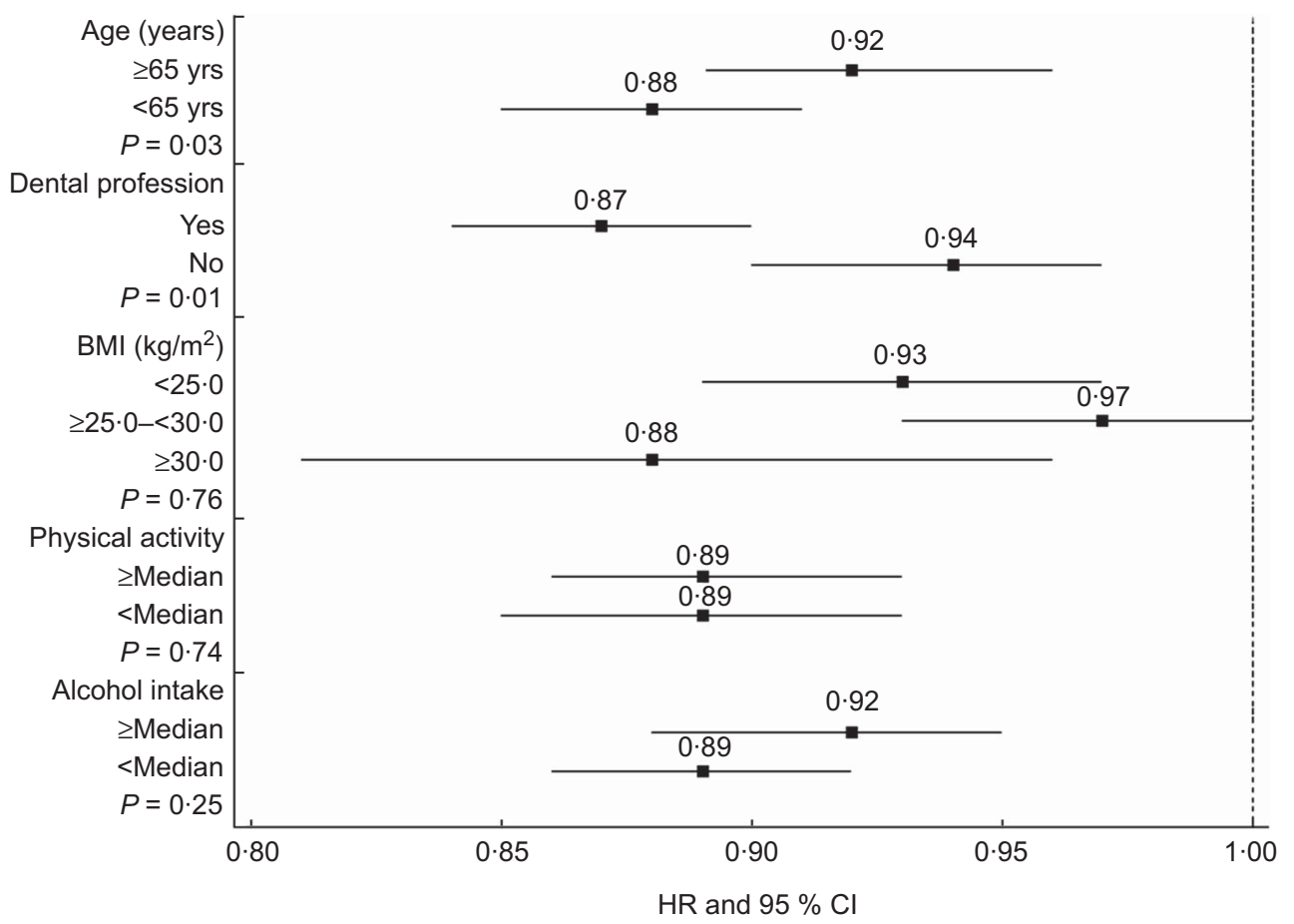

Fig. 1 Stratified multivariable-adjusted hazard ratios (HR; $\mathbf{\square}$ ) and $95 \%$ confidence intervals (represented by horizontal bars) for a $10 \mathrm{nmol} / \mathrm{l}$ increment in predicted $25(\mathrm{OH}) \mathrm{D}$ score* and tooth loss, according to categories of age, dental profession, BMI, physical activity and alcohol intake, among 42730 men participating in the Health Professionals Follow-up Study, USA, $1986-2006$. $P$ values shown are for interaction based on likelihood ratio test; - - - shows null effect $(25(\mathrm{OH}) \mathrm{D}, 25$-hydroxyvitamin $\mathrm{D})$

Tooth loss is most commonly due to caries and/or periodontitis. There is a paucity of data evaluating the associations between $25(\mathrm{OH}) \mathrm{D}$ concentrations, tooth loss and periodontitis. Furthermore, most studies have been cross-sectional, obfuscating the temporal sequence. One study evaluated the association between measures of vitamin $\mathrm{D}$, periodontitis and tooth loss and four others examined vitamin D status and periodontitis exclusively; results have been inconsistent. Krall et al. ${ }^{(28)}$ reported that randomization to $\mathrm{Ca}$ and vitamin $\mathrm{D}$ supplements resulted in a $60 \%$ lower odds of tooth loss compared with placebo $(\mathrm{OR}=0 \cdot 40 ; 95 \% \mathrm{CI} 0 \cdot 20,0 \cdot 90)$, yet no association was observed with history of periodontal treatment; however, individual effects could not be established. On the other hand, exposure to sunlight, rather than diet, constitutes the primary contribution to serum vitamin D among non-institutionalized individuals ${ }^{(7,29)}$. Three other studies $^{(5,30,31)}$ reported low $25(\mathrm{OH}) \mathrm{D}$ concentrations were associated with higher prevalence of periodontitis, while another study observed higher concentrations were associated with higher prevalence of disease ${ }^{(32)}$.

There is a paucity of data available with respect to the association between vitamin D and risk of caries. Dunning ${ }^{(33)}$ used geographical variation/latitude as a surrogate marker of $25(\mathrm{OH}) \mathrm{D}$ due to UV-B exposure among World War I to World War II Army and Navy recruits ${ }^{(34-36)}$. Results were suggestive of a higher prevalence of caries associated with greater latitude. Vitamin D may influence caries risk through its effects on innate antimicrobial peptides such as cathelicidin; however, data are limited ${ }^{(37)}$.

Vitamin D may influence tooth loss through periodontitis via immunomodulatory or antimicrobial effects, Ca absorption and/or effects on bone metabolism. Various cell types express vitamin $\mathrm{D}$ receptors, including immune cells, and are capable of converting 25(OH)D to the metabolically active 1,25 -dihydroxyvitamin $\mathrm{D}\left(1,25(\mathrm{OH})_{2} \mathrm{D}\right)^{(38)}$. When ample plasma 25(OH)D substrate is available for $1,25(\mathrm{OH})_{2} \mathrm{D}$ production, local production of $1,25(\mathrm{OH})_{2} \mathrm{D}$ by various immune cells may play a key role in regulating the innate or acquired immune response at local sites of inflammation ${ }^{(39)}$. The pro-inflammatory cytokines IL-1 $\beta$ and TNF- $\alpha$ both play central roles in the pathogenesis of periodontitis by impairing wound healing and inducing bone resorption ${ }^{(40)} \cdot 1,25(\mathrm{OH})_{2} \mathrm{D}$ has been shown to inhibit monocyte production of IL- $1 \beta$ and TNF- $\alpha$ both in vitro and in vivo ${ }^{(41-43)}$, supporting a role for locally produced $1,25(\mathrm{OH})_{2} \mathrm{D}$ in the regulation of periodontal health.

The observed association between the predicted $25(\mathrm{OH}) \mathrm{D}$ score and tooth loss could be mediated through periodontitis, caries or both. If the association was primarily mediated through periodontitis, one would expect a stronger association between the predicted 25(OH)D score and periodontitis than with tooth loss. Hence, similar associations observed with both outcomes may be suggestive of alternative pathways in addition to periodontitis. While self-reported periodontitis has previously 
Table 3 Multivariable association between predicted 25(OH)D score components and tooth loss* among 42730 men participating in the Health Professionals Follow-up Study, USA, 1986-2006

\begin{tabular}{|c|c|c|c|c|}
\hline Variable & $\mathrm{HRt}$ & $95 \% \mathrm{Cl}$ & $P$ value & $P$ value for trend \\
\hline \multicolumn{5}{|l|}{ UV-B flux (J/cm²) } \\
\hline$<461$ (ref.) & 1.00 & - & & $<0.0001$ \\
\hline $461-<628$ & 0.93 & $0.90,0.97$ & 0.001 & \\
\hline$\geq 628$ & 0.86 & $0.82,0.90$ & $<0.0001$ & \\
\hline \multicolumn{5}{|l|}{$\mathrm{BMI}\left(\mathrm{kg} / \mathrm{m}^{2}\right)$} \\
\hline$<22 \cdot 0$ (ref.) & $1 \cdot 00$ & - & & $<0.0001$ \\
\hline $22 \cdot 0-24 \cdot 9$ & 0.99 & $0.92,1.05$ & 0.68 & \\
\hline $25 \cdot 0-29 \cdot 9$ & $1 \cdot 10$ & $1 \cdot 03,1 \cdot 18$ & $<0.01$ & \\
\hline $30 \cdot 0-34 \cdot 9$ & $1 \cdot 28$ & $1 \cdot 18,1 \cdot 39$ & $<0.0001$ & \\
\hline$\geq 35 \cdot 0$ & $1 \cdot 58$ & $1 \cdot 39,1 \cdot 79$ & $<0.0001$ & \\
\hline \multicolumn{5}{|c|}{ Physical activity (MET/week) } \\
\hline Q5 (ref.) & $1 \cdot 00$ & - & & 0.01 \\
\hline Q4 & 0.99 & $0.93,1.04$ & 0.62 & \\
\hline Q3 & 0.99 & $0.93,1.04$ & 0.63 & \\
\hline Q2 & $1 \cdot 02$ & $0.96,1 \cdot 07$ & 0.59 & \\
\hline Q1 & 1.07 & $1 \cdot 01,1 \cdot 13$ & 0.03 & \\
\hline \multicolumn{5}{|l|}{ Race } \\
\hline Caucasian (ref.) & $1 \cdot 00$ & - & & \\
\hline African American & $1 \cdot 17$ & $0 \cdot 98,1 \cdot 40$ & 0.08 & \\
\hline Asian & $1 \cdot 21$ & $1 \cdot 06,1 \cdot 38$ & 0.004 & \\
\hline Other & 0.99 & $0.90,1.09$ & 0.85 & \\
\hline \multicolumn{5}{|c|}{ Vitamin D from food $(\mu \mathrm{g} / \mathrm{d})$} \\
\hline$\geq 10 \cdot 00$ (ref.) & $1 \cdot 00$ & - & & 0.98 \\
\hline $7 \cdot 50-9 \cdot 99$ & 0.94 & $0 \cdot 89,1 \cdot 00$ & 0.05 & \\
\hline $5 \cdot 00-7 \cdot 49$ & 0.95 & $0.90,1.00$ & 0.05 & \\
\hline $2 \cdot 50-4.99$ & 0.98 & $0 \cdot 93,1 \cdot 04$ & 0.56 & \\
\hline$<2 \cdot 50$ & 0.97 & $0 \cdot 89,1 \cdot 04$ & 0.39 & \\
\hline \multicolumn{5}{|c|}{ Vitamin D from supplements $(\mu \mathrm{g} / \mathrm{d})$} \\
\hline$\geq 10.00$ (ref.) & $1 \cdot 00$ & - & & $0 \cdot 20$ \\
\hline $5 \cdot 00-9 \cdot 99$ & 0.92 & $0.87,0.98$ & 0.01 & \\
\hline $0.025-4.99$ & 0.91 & $0.85,0.97$ & 0.003 & \\
\hline 0 & 0.96 & $0.90,1.02$ & $0 \cdot 19$ & \\
\hline
\end{tabular}

25(OH)D, 25-hydroxyvitamin D; HR, hazard ratio; Q5-Q1, quintile 5-quintile 1; ref., reference category; MET, metabolic equivalents. *Predicted 25(OH)D score not included in model.

tModel adjusted for all predictor score covariates presented in the model including smoking (CSI, comprehensive smoking index), pipe (yes/no), chewing tobacco (yes/no), multivitamin use (yes/no), vitamin E (quintiles), vitamin C (quintiles), dental profession (dentist/non-dentist), alcohol consumption $(0,0 \cdot 1-4 \cdot 9,5 \cdot 0-14 \cdot 9,15 \cdot 0-29 \cdot 9, \geq 30 \cdot 0 \mathrm{~g} / \mathrm{d})$, routine physical examination (yes/no) and diabetes (yes $/ \mathrm{no})$.

shown good validity in this population, it may be subject to greater misclassification than the tooth loss measure, potentially explaining the observed attenuation of periodontitis estimates, although this could not be assessed. Furthermore, we cannot account for severity of disease which may attenuate results. To quantify misclassification of the outcome, an adjusted HR was estimated from the $\log$ of the uncorrected effect estimate divided by the sum of the mean predictive values among dentists and non-dentists and $-1^{(27)}$. The multivariable-adjusted HR estimate for the highest quintile of the predicted $25(\mathrm{OH}) \mathrm{D}$ score compared with the lowest from Table 2 (Model 1) was $0.65 v$. an uncorrected estimate of $0 \cdot 80$. Additionally, although information on periodontal treatment is unavailable, the level of care is expected to be high.

We additionally evaluated the impact of the predicted $25(\mathrm{OH}) \mathrm{D}$ score components on risk of tooth loss and periodontitis. First, the association between the predicted $25(\mathrm{OH}) \mathrm{D}$ score and periodontitis was substantially attenuated upon adjustment for three key components (BMI, race and physical activity), unlike the association for tooth loss, which was attenuated only by including BMI.
Second, when modelling the associations between the predicted 25(OH)D score components and tooth loss without the predicted $25(\mathrm{OH}) \mathrm{D}$ score itself, dietary sources of vitamin D were not associated with tooth loss, while the strongest associations were observed for exposure to UV-B radiation and BMI; similar results were observed for periodontitis. These results may suggest non-25(OH)D related pathways or alternative exposure windows, which are not well understood (UV-B in childhood $v$. exposure proximate to the pathogenesis of disease). Further, confounding by socio-cultural factors related to region and oral disease may be intractable. Additionally, increased adiposity may influence periodontitis by inducing low-grade inflammation and/or immune cell dysfunction related to dyslipidaemia; data for caries are equivocal $^{(44)}$. Adjustment for Ca did not change the estimates, suggesting associations independent of Ca absorption.

We conducted several a priori sub-analyses and observed that the association between the predicted $25(\mathrm{OH}) \mathrm{D}$ score and tooth loss varied by age and dental profession. While plausible that these observations were due to chance, one may consider alternative explanations. 
Age-related tooth loss is multifactorial, hence tooth loss at younger ages may reflect specific pathology potentially mediated in part by $25(\mathrm{OH}) \mathrm{D}$-impaired immune response. Although highly speculative, the variation by dental profession may reflect different underlying susceptibilities to tooth loss, periodontitis and/or caries between dental and non-dental professionals due to oral health behaviours. Oral disease-related tooth loss among non-dental health professionals may largely reflect the effects of plaque and other localized risk factors; whereas, among those with a higher level of dental care (e.g. dental professionals), the pathogenesis may reflect a greater susceptibility to oral disease by systemic factors including but not limited to plasma $25(\mathrm{OH}) \mathrm{D}$.

Although the present study consisted primarily of white men, similar results have been reported in diverse populations. It is unlikely that residual confounding or an unmeasured factor would entirely account for the observed associations given the longitudinal assessment of potential confounders and modest attenuation towards the null between the age- and multivariable-adjusted models.

Important strengths of the study include the use of a novel prediction score which does not replace plasma measurements, but has been shown to predict approximately a $10 \mathrm{ng} / \mathrm{ml}$ difference in true $25(\mathrm{OH}) \mathrm{D}$ at extremes, representing a physiologically important increment ${ }^{(45)}$. However, measurement error in the predicted $25(\mathrm{OH}) \mathrm{D}$ score may mask any underlying threshold effect in the exposure disease association as linear. Furthermore, study power was increased by use of the full cohort irrespective of biomarker samples. Additional strengths include the longitudinal design with 20 years of follow-up including updated values of covariates and a homogeneous population minimizing confounding by socio-economic status and health-oriented behaviours.

\section{Conclusion}

The present results suggest that vitamin D itself and/or components associated with vitamin D status including UV-B may be associated with lower risk of tooth loss and periodontitis. These results warrant further investigation by epidemiological and clinical studies. Since vitamin D can be obtained free or at low cost, the implications of these associations in primary prevention of periodontitis and tooth loss are large.

\section{Acknowledgements}

Sources of funding: The study was supported by grant R03DE017948 from the National Institute of Dental and Craniofacial Research of the National Institutes of Health. The authors wish to acknowledge institutional support from the Channing Division of Network Medicine, Department of Medicine, Brigham and Women's Hospital and Harvard
Medical School. Conflicts of interest: The authors declare they have no conflicts of interest. Authors' contributions: T.D., E.G. and E.K.K. designed the research; K.J.J. contributed to designing the oral health data collection; M.J., T.D. and E.G. conducted the research; M.J. analysed the data; M.J., T.D., E.G. and E.K.K. interpreted findings; M.J., K.J.J., E.G. and T.D. contributed to writing the paper; and T.D. had primary responsibility for final content. All authors read and approved the final manuscript.

\section{References}

1. Dawson-Hughes B, Heaney RP, Holick MF et al. (2005) Estimates of optimal vitamin D status. Osteoporos Int 16, 713-716

2. Giovannucci E (2005) The epidemiology of vitamin D and cancer incidence and mortality: a review (United States). Cancer Causes Control 16, 83-95.

3. Heaney RP (2005) The vitamin D requirement in health and disease. J Steroid Biochem Mol Biol 97, 13-19.

4. Pittas AG, Lau J, Hu FB et al. (2007) The role of vitamin D and calcium in type 2 diabetes. A systematic review and meta-analysis. J Clin Endocrinol Metab 92, 2017-2029.

5. Dietrich T, Joshipura KJ, Dawson-Hughes B et al. (2004) Association between serum concentrations of 25-hydroxyvitamin $\mathrm{D}_{3}$ and periodontal disease in the US population. Am J Clin Nutr 80, 108-113.

6. Dietrich T, Nunn M, Dawson-Hughes B et al. (2005) Association between serum concentrations of 25-hydroxyvitamin D and gingival inflammation. Am J Clin Nutr 82, $575-580$.

7. Newton HM, Sheltawy M, Hay AW et al. (1985) The relations between vitamin $\mathrm{D}_{2}$ and $\mathrm{D}_{3}$ in the diet and plasma $25 \mathrm{OHD}_{2}$ and $25 \mathrm{OHD}_{3}$ in elderly women in Great Britain. Am J Clin Nutr 41, 760-764.

8. Nesby-O'Dell S, Scanlon KS, Cogswell ME et al. (2002) Hypovitaminosis D prevalence and determinants among African American and white women of reproductive age: third National Health and Nutrition Examination Survey, 1988-1994. Am J Clin Nutr 76, 187-192.

9. Looker AC, Dawson-Hughes B, Calvo MS et al. (2002) Serum 25-hydroxyvitamin D status of adolescents and adults in two seasonal subpopulations from NHANES III. Bone 30, 771-777.

10. Flegal KM, Carroll MD, Ogden CL et al. (2010) Prevalence and trends in obesity among US adults, 1999-2008. JAMA 303, 235-241.

11. Wortsman J, Matsuoka LY, Chen TC et al. (2000) Decreased bioavailability of vitamin D in obesity. Am J Clin Nutr $\mathbf{7 2}$, 690-693.

12. Rimm EB, Stampfer MJ, Colditz GA et al. (1990) Effectiveness of various mailing strategies among nonrespondents in a prospective cohort study. Am J Epidemiol 131, 1068-1071.

13. Rimm EB, Stampfer MJ, Giovannucci E et al. (1995) Body size and fat distribution as predictors of coronary heart disease among middle-aged and older US men. Am J Epidemiol 141, 1117-1127.

14. Dietrich T \& Hoffmann K (2004) A comprehensive index for the modeling of smoking history in periodontal research. J Dent Res $\mathbf{8 3}, 859-863$.

15. Leffondre K, Abrahamowicz M, Xiao Y et al. (2006) Modelling smoking history using a comprehensive smoking index: application to lung cancer. Stat Med 25, 4132-4146.

16. Rimm EB, Giovannucci EL, Stampfer MJ et al. (1992) Reproducibility and validity of an expanded self-administered semiquantitative food frequency questionnaire among male health professionals. Am J Epidemiol 135, 1114-1126. 
17. Giovannucci E, Colditz G, Stampfer MJ et al. (1991) The assessment of alcohol consumption by a simple selfadministered questionnaire. Am J Epidemiol 133, 810-817.

18. Hu FB, Leitzmann MF, Stampfer MJ et al. (2001) Physical activity and television watching in relation to risk for type 2 diabetes mellitus in men. Arch Intern Med 161, $1542-1548$

19. Chasan-Taber S, Rimm EB, Stampfer MJ et al. (1996) Reproducibility and validity of a self-administered physical activity questionnaire for male health professionals. Epidemiology 7, 81-86.

20. Giovannucci E, Liu Y, Rimm EB et al. (2006) Prospective study of predictors of vitamin D status and cancer incidence and mortality in men. J Natl Cancer Inst 98, 451-459.

21. Scotto J, Fears T \& Faumeni JJ (1996) Solar radiation. In Cancer Epidemiology and Prevention, pp. 355-372 [D Schotenfeld and JJ Fraumeni, editors]. New York: Oxford University Press.

22. Douglass CW, Berlin J \& Tennstedt S (1991) The validity of self-reported oral health status in the elderly. $J$ Public Health Dent 51, 220-222.

23. Joshipura KJ, Pitiphat W \& Douglass CW (2002) Validation of self-reported periodontal measures among health professionals. J Public Health Dent 62, 115-121.

24. Joshipura KJ, Douglass CW, Garcia RI et al. (1996) Validity of a self-reported periodontal disease measure. $J$ Public Health Dent 56, 205-212.

25. Reddy MS (1997) The use of periodontal probes and radiographs in clinical trials of diagnostic tests. Ann Periodontol 2, 113-122.

26. Cox DR (1972) Regression models and life-tables. $J$ R Stat Soc Ser B Method 34, 187-220.

27. Duffy SW, Warwick J, Williams AR et al. (2004) A simple model for potential use with a misclassified binary outcome in epidemiology. J Epidemiol Community Health 58, 712-717.

28. Krall EA, Wehler C, Garcia RI et al. (2001) Calcium and vitamin D supplements reduce tooth loss in the elderly. Am J Med 111, 452-456.

29. Lips P, van Ginkel FC, Jongen MJ et al. (1987) Determinants of vitamin D status in patients with hip fracture and in elderly control subjects. Am J Clin Nutr 46, 1005-1010.

30. Miley DD, Garcia MN, Hildebolt CF et al. (2009) Crosssectional study of vitamin D and calcium supplementation effects on chronic periodontitis. J Periodontol 80, 1433-1439.
31. Garcia MN, Hildebolt CF, Miley DD et al. (2011) One-year effects of vitamin D and calcium supplementation on chronic periodontitis. J Periodontol 82, 25-32.

32. Liu K, Meng H, Tang X et al. (2009) Elevated plasma calcifediol is associated with aggressive periodontitis. J Periodontol 80, 1114-1120.

33. Dunning JM (1953) The influence of latitude and distance from seacoast on dental disease. J Dent Res 32, 811-829.

34. Britten RH \& Perott GSJ (1941) Summary of physical findings on men drafted in the World War. Public Health Rep 56, 41-62.

35. Ferguson RA (1935) Some observations on diet and dental disease. J Am Dent Assoc 22, 392-401.

36. Nizel AE \& Bibby BG (1944) Geographical variations in caries prevalence in soldiers. J Am Dent Assoc 31, 1619-1626.

37. Park K, Elias PM, Oda Y et al. (2011) Regulation of cathelicidin expression by an ER stress-signaling, vitamin D receptor-independent pathway. J Biol Chem 286, 34121-34130.

38. Zittermann A (2003) Vitamin D in preventive medicine: are we ignoring the evidence? Br J Nutr 89, 552-572.

39. Adams JS, Liu PT, Chun R et al. (2007) Vitamin D in defense of the human immune response. Ann N Y Acad Sci 1117, 94-105.

40. Kornman KS, Page RC \& Tonetti MS (1997) The host response to the microbial challenge in periodontitis: assembling the players. Periodontol 2000 14, 33-53.

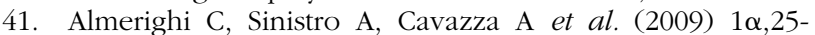
Dihydroxyvitamin $\mathrm{D}_{3}$ inhibits CD40L-induced proinflammatory and immunomodulatory activity in human monocytes. Cytokine 45, 190-197.

42. Muller K, Gram J, Bollerslev J et al. (1991) Down-regulation of monocyte functions by treatment of healthy adults with $1 \alpha, 25$-dihydroxyvitamin $\mathrm{D}_{3}$. Int J Immunopharmacol 13, 525-530.

43. Muller K, Haahr PM, Diamant M et al. (1992) 1,25Dihydroxyvitamin $\mathrm{D}_{3}$ inhibits cytokine production by human blood monocytes at the post-transcriptional level. Cytokine 4, 506-512.

44. Chaffee BW \& Weston SJ (2010) Association between chronic periodontal disease and obesity: a systematic review and meta-analysis. J Periodontol 81, 1708-1724.

45. Bertrand KA, Giovannucci E, Liu Y et al. (2012) Determinants of plasma 25-hydroxyvitamin D and development of prediction models in three US cohorts. Br J Nutr $\mathbf{1 0 8}$ 1889-1896. 